\title{
Learning Directed Probabilistic Logical Models: Ordering-Search Versus Structure-Search
}

\author{
Daan Fierens, Jan Ramon, Maurice Bruynooghe, and Hendrik Blockeel
}

\author{
K.U.Leuven, Dept. of Computer Science, Celestijnenlaan 200A, 3001 Heverlee, Belgium \\ \{Daan.Fierens, Jan. Ramon, Maurice. Bruynooghe, \\ Hendrik.Blockeel\}@cs. kuleuven. be
}

\begin{abstract}
We discuss how to learn non-recursive directed probabilistic logical models from relational data. This problem has been tackled before by upgrading the structure-search algorithm initially proposed for Bayesian networks. In this paper we propose to upgrade another algorithm, namely ordering-search, since for Bayesian networks this was found to work better than structure-search. We experimentally compare the two upgraded algorithms on two relational domains. We conclude that there is no significant difference between the two algorithms in terms of quality of the learnt models while ordering-search is significantly faster.
\end{abstract}

Keywords: statistical relational learning, probabilistic logical models, inductive logic programming, Bayesian networks, probability trees, structure learning.

\section{Introduction}

A Bayesian network is a compact specification of a joint probability distribution on a set of random variables under the form of a directed acyclic graph (the structure) and a set of conditional probability distributions (CPDs). When learning from data the goal is usually to find the structure and CPDs that maximize a certain scoring criterion. The most traditional approach to learning Bayesian networks is structure-search [5]. Recently, an alternative algorithm called ordering-search was introduced that was found to perform at least as good as structure-search while usually being faster [9].

The past few years a variety of formalisms has been introduced to describe probabilistic logical models. Many of these formalisms deal with directed models that are upgrades of Bayesian networks to the relational case. Learning algorithms have been developed for several such formalisms [4677. Most of these algorithms are essentially upgrades of the structure-search algorithm for Bayesian networks. In this paper we investigate how ordering-search can be upgraded to the relational case.

The contributions of this paper are two-fold. First, we upgrade the ordering-search algorithm towards learning non-recursive directed probabilistic logical models. Second, we experimentally compare the resulting algorithm with the upgraded structure-search algorithm on two relational domains. We use the formalism Logical Bayesian Networks but the proposed approach is also valid for related formalisms such as Probabilistic Relational Models, Bayesian Logic Programs and Relational Bayesian Networks [1].

This paper is structured as follows. We review Logical Bayesian Networks in Section 2 . We discuss learning in Section 3 and show experimental results in Section 4 . We conclude in Section 5 More details about Sections 2 to 4 are given in the full paper [3]. 


\section{Logical Bayesian Networks}

We now briefly review Logical Bayesian Networks. For details and formal semantics we refer to the full papers [13]. A Logical Bayesian Network or LBN is essentially a specification of a Bayesian network conditioned on some logical input predicates describing the domain of discourse. For instance, when modelling the well-known 'university' domain [4], we would use predicates student/1, course/1, prof $/ 1$, teaches $/ 2$ and takes $/ 2$ with their obvious meanings. The semantics of an LBN is that, given an interpretation of these logical predicates, the LBN induces a particular Bayesian network.

In LBNs random variables are represented as ground atoms built from certain special predicates, the probabilistic predicates. For instance, if intelligence/1 is a probabilistic predicate then the atom intelligence(ann) is called a probabilistic atom and represents a random variable. Which random variables exist for a particular interpretation of the logical predicates is determined by a set of random variable declarations [1]. An LBN contains two parts that are typically to be learned: a set of dependency clauses and a set of logical CPDs. We now illustrate this for the university domain.

The dependency clauses for the university domain are the following.

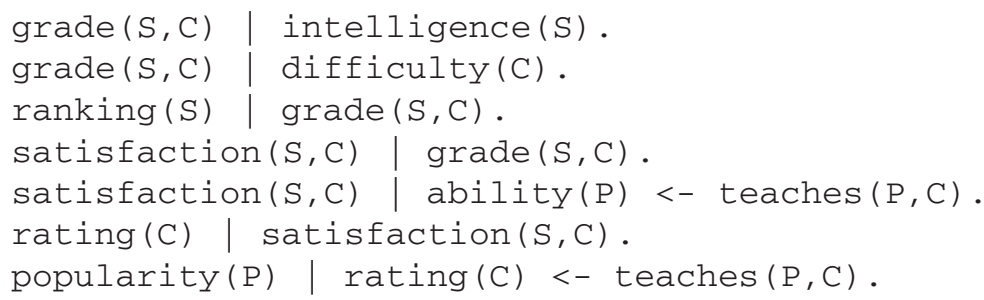

Informally, the first clause should be read as "the grade of a student $S$ for a course $C$ depends on the intelligence of $S$ " and the last clause as "the popularity of a professor $P$ depends on the rating of a course $C$ if $P$ teaches $C$ ". In this clause, popularity $(P)$ is called the head, rating $(C)$ the body and teaches $(P, C)$ the context of the clause.

To quantify the dependencies specified by the dependency clauses, LBNs associate with each probabilistic predicate a so-called logical CPD. In this work we represent logical CPDs under the form of logical probability trees [2]. The internal nodes in the tree for a probabilistic atom $p_{\text {target }}$ can contain a) tests on the values of probabilistic atoms that are parents of $p_{\text {target }}$ according to the dependency clauses, b) conjunctions of logical literals, and c) combinations of the two. Leaves contain probability distributions on the values of $p_{\text {target }}$. An example of such a tree is shown in Figure 1.

\section{Learning Non-recursive Logical Bayesian Networks}

The dependency clauses and the logical CPDs in an LBN can be learned from a dataset of examples where each example consists of two parts: an interpretation of the logical predicates and an assignment of values to all ground random variables (as determined by the random variable declarations). The goal of learning is to find the clauses and logical CPDs that maximize the scoring criterion. In this paper we only deal with learning nonrecursive $\mathrm{LBNs}$, i.e. LBNs with non-recursive dependency clauses. For a discussion on the relation to learning recursive LBNs we refer to the full paper [3]. 


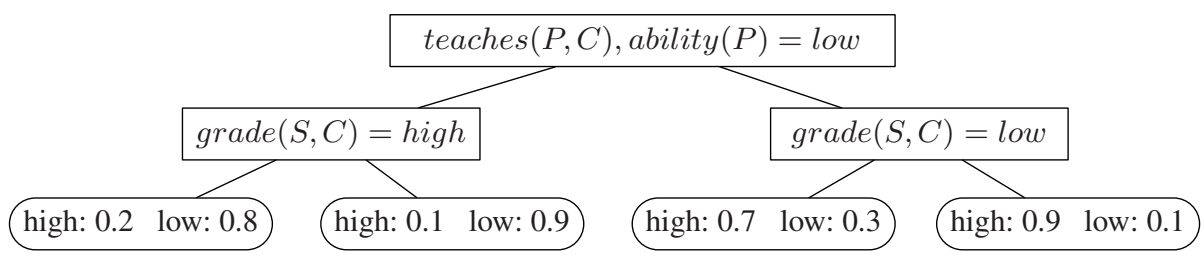

Fig. 1. Example of a logical CPD for satisfaction $(S, C)$. Tests in internal nodes are binary. When a test succeeds the left branch is taken, when it fails the right branch is taken.

Next we discuss a generic hillclimbing algorithm for learning non-recursive LBNs and give two instantiations of this generic algorithm. The first is the upgraded orderingsearch algorithm that we introduce in this paper. The second is the existing upgraded structure-search algorithm. To stress the difference with Logical Bayesian Networks we will refer to ordinary Bayesian networks as 'propositional' Bayesian networks.

\subsection{A Generic Hillclimbing Algorithm for Learning Logical Bayesian Networks}

For LBNs, as for propositional Bayesian networks, there exists a generic learning algorithm of which the structure-search and ordering-search algorithms are specific instantiations. The idea is to perform hillclimbing-search through a space of solutions. In the case of structure-search a solution is a structure (a set of dependency clauses), while in the case of ordering-search it is an ordering on the set of probabilistic predicates. The generic algorithm is shown in Figure 2. The neighbourhood of the current solution is the set of solutions that can be obtained by making a small change to the current solution. The score of a solution is computed as the product of the scores of the logical CPDs for that solution. In this work we learn logical CPDs under the form of logical probability trees (like the tree in Figure 1). Which probabilistic predicates can be used as input for the tree is determined by the particular solution. We use the standard probability tree algorithms in the TILDE learner and score trees using the Bayesian Information Criterion (BIC) for logical probability trees [2]. Since BIC is a decomposable scoring criterion, the above algorithm can be implemented quite efficiently [35].

To use the generic algorithm of Figure 2 with a specific kind of solutions (structures, orderings, ... ) one has to determine a) how to obtain an initial random solution 1, b) how to define the neighbourhood of a solution, and c) how to extract the dependency clauses from the learned solution. We will explain each of these issues for ordering-search in Section 3.2 and for structure-search in Section 3.3

\subsection{Ordering-Search}

First we discuss the propositional case, then we discuss case of LBNs.

\footnotetext{
${ }^{1}$ The initial solution might influence the final result since the algorithm only converges to a local optimum. Hence, we experimented with multiple runs with different initial solutions but we found this not to be significantly better than a single run.
} 


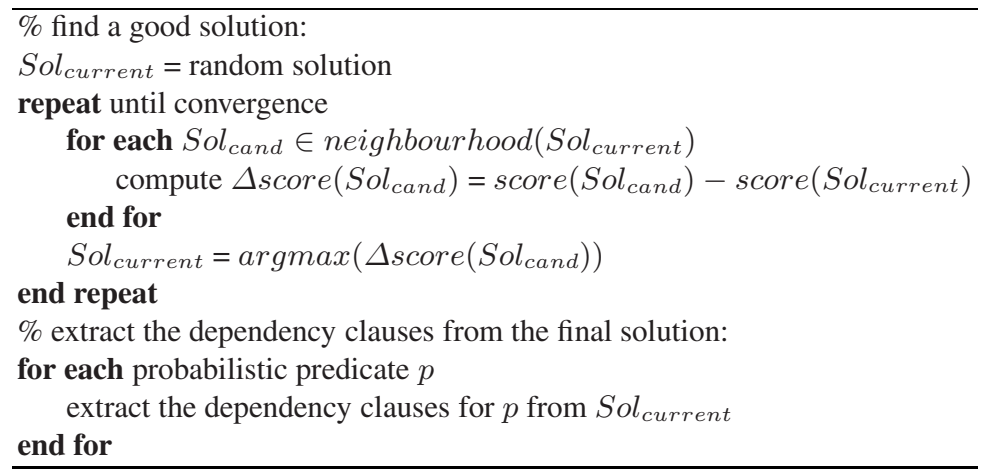

Fig. 2. Generic hillclimbing algorithm for learning LBNs. In the two instantiations of this generic algorithm that we consider, a solution $\mathrm{Sol}$ corresponds to respectively a structure or an ordering.

Ordering-Search for Propositional Bayesian Networks. Ordering-search is based on the fact that it is relatively easy to learn a Bayesian network if an ordering on the set of random variables is given [9]. Such an ordering eliminates the possibility of cycles. This makes it possible to decide for each variable $X$ separately which variables, from all variables preceding it in the ordering, are its parents. This can simply be done by learning a CPD for $X$ under the assumption that 'selective' CPDs are used, i.e. CPDs that select from all candidate inputs the relevant inputs (for instance conditional probability tables with a bound on the number of effective inputs [9]). However, the score of the Bayesian network that is learned in this way depends heavily on the quality of the ordering that is used. Hence, the idea of ordering-search is to perform hillclimbing through the space of possible orderings, in each step applying the above procedure.

Teyssier and Koller [9] experimentally compared structure-search and orderingsearch and found that ordering-search is always at least as good as structure-search and usually faster. As an explanation they note that the space of orderings is smaller than the space of structures and that ordering-search has no costly acyclicity checks.

Ordering-Search for Logical Bayesian Networks. Until now ordering-search has not yet been upgraded to the case of non-recursive directed probabilistic logical models. The above conclusions from the propositional case motivated us to investigate this. We now show how to upgrade ordering-search towards learning non-recursive LBNs.

Similar to the case of propositional Bayesian networks, it is easy to learn an LBN if an ordering on the set of probabilistic predicates is given. We can then learn an LBN simply by learning for each probabilistic predicate $p$ a logical probability tree with as inputs all predicates preceding $p$ in the ordering. Ordering-search corresponds to applying the generic algorithm of Figure 2 with orderings as solutions, this is basically hillclimbing-search through the space of orderings. The neighbourhood of an ordering $O$ is defined as the set of orderings that can be obtained by swapping a pair of adjacent predicates in $O$. As an initial ordering we use a random ordering.

Apart from using orderings on the set of predicates instead of on the set of random variables, there are two major differences between our algorithm and the propositional 
algorithm. First, we use logical probability trees instead of simple conditional probability tables. Second, in the case of LBNs once we found the optimal ordering and the logical CPDs for this ordering, we still need an extra step to extract the dependency clauses from these logical CPDs. We now discuss the latter in more detail.

Extracting the Dependency Clauses from the Logical CPDs. Below we explain how to extract the clauses from a logical probability tree. The goal is to find the most specific set of clauses that is 'consistent' with the tree [3]. To obtain an LBN, this procedure has to be applied to the probability tree for each probabilistic predicate.

When extracting clauses from a tree, we create a clause for each test on a probabilistic atom in a node of the tree. Call the atom that is tested $p_{\text {test }}$, the node $N$ and the target atom of the tree $p_{\text {target }}$. In the most general case, apart from the test on $p_{\text {test }}$, the node $N$ can contain a number of tests on other probabilistic atoms and a conjunction of logical literals. Call this conjunction $l$. We then create a clause of the form $p_{\text {target }}$ $p_{\text {test }} \leftarrow l$, path $(N)$, where path $(N)$ is a conjunction of logical literals that describes the path from the root to $N$. Each node on this path can contribute a number of logical literals to path $(N)$. A succeeded node contributes all logical literals that it contains. A failed node that does not contain any tests on probabilistic atoms contributes the negation of all its logical literals. All other failed nodes do not contribute to the path.

As an example, consider the probability tree in Figure 1. For this tree, $p_{\text {target }}$ is satisfaction $(S, C)$. For the root node, $p_{\text {test }}$ is $\operatorname{ability}(P), l$ is teaches $(P, C)$ and the path is empty. For the internal node below the root to the left, $p_{\text {test }}$ is $\operatorname{grade}(S, C), l$ is empty and the path is teaches $(P, C)$. For the node below the root to the right, $p_{\text {test }}$ is $\operatorname{grade}(S, C)$ and $l$ and the path are both empty. The three resulting clauses for these nodes are respectively the following.

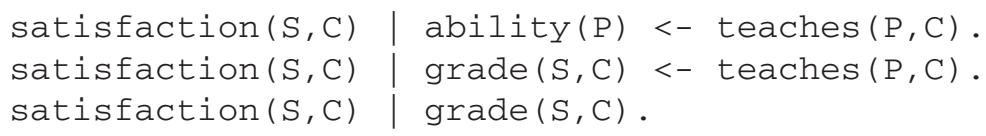

The second clause is redundant (it is a special case of the third) and can be dropped.

\subsection{Structure-Search}

Structure-search is the most traditional and most straightforward approach for learning propositional Bayesian networks [5]. It is essentially hillclimbing through the space of possible structures. The neighbourhood of the current structure typically consists of all acyclic structures that can be obtained by adding, deleting or reversing an edge.

The structure-search algorithm for propositional Bayesian networks has already been upgraded to the relational case for several formalisms [467]. The algorithm that we use for LBNs is very similar to these existing upgrades. The main difference is that we use logical probability trees instead of combining rules [67] or aggregates [4].

To derive a concrete structure-search algorithm for LBNs from the generic algorithm of Figure 2, we have to define the notion of a neighbourhood and define how an initial structure is obtained (note that the final step in the generic algorithm, extracting clauses from the solution, is not needed for structure-search since a solution is already a set of dependency clauses). We define the neighbourhood of the current structure as the 
set of all non-recursive structures that can be obtained by adding a dependency clause, deleting a clause or swapping the head and body of a clause in the current structure. To find an initial set of clauses we borrow some elements from the ordering-search algorithm. Specifically, we generate a random ordering, learn logical CPDs for this ordering and apply the procedure for extracting dependency clauses from logical CPDs. As a consequence, in our experiments ordering-search and structure-search always start from the same point. This ensures that an experimental comparison of both algorithms effectively evaluates the search process itself and not the starting point of the search.

\section{Experiments}

We first discuss the datasets and the experimental setup. Then we discuss our results.

\subsection{Datasets and Experimental Setup}

We perform experiments on two relational domains. First, we generate synthetic datasets of varying size from the given LBN for the university domain (see Section 2). We use 5, $10,15,31,62,125$ and 250 examples. Each example corresponds to 230 random variables (describing 20 students, 10 courses and 5 professors) and examples are mutually independent. Second, we use the UWCSE dataset, a real-world dataset constructed by extracting information about graduate students, professors and courses from the web pages of a computer science department [8]. Since in this dataset relations are of special importance 2 , we model them as probabilistic predicates, leading to what Getoor et al. call "relational uncertainty" [4]. This dataset consists of 5 disjoint subsets (each corresponding to a specific research area) and contains 9607 random variables in total (describing 140 students, 132 courses and 52 professors).

For all experiments we performed 5-fold cross validation. For the university domain, examples were assigned to folds randomly. For UWCSE, each fold corresponds to a research area. We report the average results over the folds and use two-tailed paired t-tests (with $\alpha=0.05$ ) to assess the significance of differences between two algorithms.

We use six evaluation criteria. The first four criteria measure characteristics of the learned LBN: normalized test log-likelihood (the log-likelihood on the test data divided by the number of examples), normalized train score (the score on the training data divided by the number of examples; while not important in itself, this gives some additional insight into the results), number of dependency clauses learned (smaller is usually better because of ease of interpretation) and number of correct dependency clauses learned (we can only report this for the synthetic university domain since there we know the correct dependency clauses). The other two criteria measure the efficiency of the learning process: runtime and average time per iteration (in terms of the generic algorithm in Figure 2. one iteration is one step in the repeat loop; while not important in itself, this time gives an idea about the size of a neighbourhood and how efficiently all elements in a neighbourhood can be scored and hence helps to explain runtime).

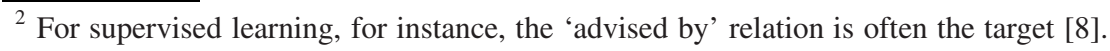




\subsection{Results}

The results for ordering-search (OS) and structure-search (SS) are shown in Table 1.

Table 1. Experimental results on various datasets. If the result for one of the two algorithms (OS or SS) is significantly better than the result for the other, the best result is indicated in bold.

\begin{tabular}{lccccccc}
\hline Dataset & Method LogLik(Test) & Score(Train) & \#Clauses \#CorrectClauses Time TimePerIter \\
\hline Univ5 & OS & -1.3789 & -1.3485 & 9.0 & 2.0 & $\mathbf{2 7 s}$ & $\mathbf{6 s}$ \\
Univ5 & SS & -1.3750 & -1.3365 & 9.8 & 3.6 & $130 \mathrm{~s}$ & $17 \mathrm{~s}$ \\
Univ5 & empty & -1.4799 & -1.4989 & - & - & - & - \\
\hline Univ10 & OS & -1.3669 & -1.3524 & 9.8 & 4.2 & $\mathbf{3 3 s}$ & $11 \mathrm{~s}$ \\
Univ10 & SS & -1.3461 & -1.3410 & 9.4 & 5.0 & $126 \mathrm{~s}$ & $20 \mathrm{~s}$ \\
Univ10 & empty & -1.4722 & -1.4880 & - & - & - & - \\
\hline Univ15 & OS & -1.3444 & -1.3415 & 8.6 & 3.2 & $\mathbf{4 2 s}$ & $\mathbf{8 s}$ \\
Univ15 & SS & -1.3328 & -1.3305 & 9.6 & $\mathbf{3 . 8}$ & $155 \mathrm{~s}$ & $20 \mathrm{~s}$ \\
Univ15 & empty & -1.4697 & -1.4792 & - & - & - & - \\
\hline Univ31 & OS & -1.3083 & -1.3135 & 8.8 & 5.0 & $\mathbf{4 3 s}$ & $\mathbf{1 2 s}$ \\
Univ31 & SS & -1.3023 & -1.3060 & 9.6 & 6.4 & $158 \mathrm{~s}$ & $24 \mathrm{~s}$ \\
Univ31 & empty & -1.4575 & -1.4647 & - & - & - & - \\
\hline Univ62 & OS & -1.3051 & -1.3097 & 11.2 & 4.8 & $\mathbf{6 3 s}$ & $\mathbf{1 3 s}$ \\
Univ62 & SS & -1.2973 & -1.3012 & 10.4 & 6.2 & $249 \mathrm{~s}$ & $30 \mathrm{~s}$ \\
Univ62 & empty & -1.4554 & -1.4595 & - & - & - & - \\
\hline Univ125 & OS & -1.2905 & -1.2959 & 8.8 & 5.0 & $\mathbf{9 9 s}$ & $\mathbf{1 9 s}$ \\
Univ125 & SS & -1.2828 & -1.2861 & 8.2 & $\mathbf{6 . 6}$ & $389 \mathrm{~s}$ & $62 \mathrm{~s}$ \\
Univ125 & empty & -1.4562 & -1.4586 & - & - & - & - \\
\hline Univ250 & OS & -1.3001 & -1.3035 & 9.8 & 4.8 & $\mathbf{1 5 4 s}$ & $62 \mathrm{~s}$ \\
Univ250 & SS & -1.2894 & -1.2913 & 7.6 & $\mathbf{6 . 4}$ & $553 \mathrm{~s}$ & $84 \mathrm{~s}$ \\
Univ250 & empty & -1.4561 & -1.4573 & - & - & - & - \\
\hline UWCSE & OS & -0.4288 & -0.3539 & 15.2 & - & $\mathbf{1 8 3 s}$ & $\mathbf{4 3 s}$ \\
UWCSE & SS & -0.4160 & $\mathbf{- 0 . 3 4 8 9}$ & 14.6 & - & $586 \mathrm{~s}$ & $93 \mathrm{~s}$ \\
UWCSE & empty & -0.4631 & -0.3961 & - & - & - & - \\
\hline & & & & & & &
\end{tabular}

Table 1 reports the test log-likelihood and train score for OS and SS and also for the 'empty LBN' as a baseline. With an 'empty LBN' we mean an LBN with no dependency clauses, this is the LBN according to which all random variables are independent. For all datasets OS and SS perform significantly better than the empty LBN on both training and test data (significance is not indicated in the table). The differences between OS and SS are small as compared to the differences with the empty LBN. For none of the datasets there is a significant difference between OS and SS on the test data (although SS is significantly better on training data for UWCSE).

For none of the datasets there is a significant difference between the number of dependency clauses learned by OS and by SS. However, the number of correct dependency clauses learned is sometimes significantly higher for SS than for OS. The fact that these differences do not lead to a significantly worse likelihood or score for OS indicates that OS learns all dependencies except the very weak ones [3]. 
On all the datasets runtime is significantly lower for OS than for SS (differences range from a factor 3.2 to 4.8 ). This is explained by the fact that also the average time per iteration is lower for OS than for SS. The latter was expected since an iteration basically corresponds to scoring all solutions in the neighbourhood of the current solution and the size of the neighbourhood is typically smaller for OS than for SS (it is linear in the number of probabilistic predicates for OS but quadratic for SS).

\section{Conclusion}

In this paper we upgraded the ordering-search algorithm for propositional Bayesian networks towards non-recursive directed probabilistic logical models. We experimentally compared the resulting algorithm with the existing upgraded structure-search algorithm on two relational domains. The results show that ordering-search is competitive with structure-search in terms of quality of the learned models but is significantly faster than structure-search. We conclude that ordering-search is a good alternative to structuresearch for learning directed probabilistic logical models.

Acknowledgements. Research supported by the Institute for the Promotion of Innovation by Science and Technology in Flanders (IWT Vlaanderen), Research FoundationFlanders (FWO Vlaanderen) and GOA 2003/08 “Inductive Knowledge Bases”.

\section{References}

1. Fierens, D., Blockeel, H., Bruynooghe, M., Ramon, J.: Logical Bayesian networks and their relation to other probabilistic logical models. In: Proceedings of the 15th International Conference on Inductive Logic Programming (ILP), pp. 121-135. Springer, Heidelberg (2005)

2. Fierens, D., Ramon, J., Blockeel, H., Bruynooghe, M.: A comparison of pruning criteria for learning trees. Technical Report CW 488, Department of Computer Science, Katholieke Universiteit Leuven (April 2007)

3. Fierens, D., Ramon, J., Bruynooghe, M., Blockeel, H.: Learning directed probabilistic logical models: ordering-search versus structure-search. Technical Report CW 490, Department of Computer Science, Katholieke Universiteit Leuven (May 2007)

4. Getoor, L., Friedman, N., Koller, D., Pfeffer, A.: Learning Probabilistic Relational Models. In: Relational Data Mining, pp. 307-334. Springer, Heidelberg (2001)

5. Heckerman, D., Geiger, D., Chickering, D.: Learning Bayesian networks: The combination of knowledge and statistical data. Machine Learning 20, 197-243 (1995)

6. Kersting, K., De Raedt, L.: Towards combining inductive logic programming and Bayesian networks. In: Proceedings of the 11th International Conference on Inductive Logic Programming (ILP), pp. 118-131. Springer, Heidelberg (2001)

7. Natarajan, S., Wong, W., Tadepalli, P.: Structure refinement in First Order Conditional Influence Language. In: Proceedings of the workshop on Open Problems in Statistical Relational Learning (SRL) (2006)

8. Richardson, M., Domingos, P.: Markov logic networks. Machine Learning 62(1-2), 107-136 (2006)

9. Teyssier, M., Koller, D.: Ordering-based search: A simple and effective algorithm for learning Bayesian networks. In: Proceedings of the 21st conference on Uncertainty in AI (UAI), pp. 584-590. AUAI Press (2005) 